\title{
COMMENTARY
}

\section{Could $\mathrm{Ca}^{2+}$ sensitizers rescue patients from chronic congestive heart failure?}

\author{
M Endoh \\ Department of Cardiovascular Pharmacology, Yamagata University School of Medicine, Yamagata, Japan
}

\begin{abstract}
Attempts to ameliorate cardiac contractile dysfunction by $\mathrm{Ca}^{2+}$ mobilizers, such as catecholamines, phosphodiesterase (PDE) inhibitors and digitalis, play an important role in pharmacotherapy for congestive heart failure (CHF), but these agents possess disadvantages in causing $\mathrm{Ca}^{2+}$ overload resulting in arrhythmogenicity and damage to cardiomyocytes. $\mathrm{Ca}^{2+}$ sensitizers that act directly on contractile proteins are free from the risk of $\mathrm{Ca}^{2+}$ overload and they could improve haemodynamic parameters with minimum increase in energy expenditure even under pathological conditions, including acidosis and stunned myocardium. Beneficial effects of levosimendan (that acts by combination of $\mathrm{Ca}^{2+}$ sensitization and PDE inhibition) on CHF due to hypertensive cardiomyopathy in Dahl/Rapp rats as reported in this issue demonstrate the potential of oral levosimendan in long-term treatment of chronic CHF. Since chronic CHF in clinical settings is much more complex, careful analysis of clinical outcomes will be required to establish the therapeutic relevance of $\mathrm{Ca}^{2+}$ sensitizers in the treatment of chronic $\mathrm{CHF}$.
\end{abstract}

British Journal of Pharmacology (2007) 150, 826-828. doi:10.1038/sj.bjp.0707163; published online 26 February 2007

Keywords: apoptosis; $\mathrm{Ca}^{2+}$ sensitizers; congestive heart failure; hypertensive Dahl/Rapp rats; levosimendan; PDE III inhibitors; remodelling

\begin{abstract}
Abbreviations: ANP, atrial natriuretic peptide; $\mathrm{BNP}$, brain natriuretic peptide; $\mathrm{CHF}$, congestive heart failure; $\mathrm{NCX} \mathrm{Na}^{+}-\mathrm{Ca}^{2+}$ exchanger; PDE, phosphodiesterase; PIE, positive inotropic effect; QOL, quality of life; SERCA, sarco/ endoplasmic reticulum $\mathrm{Ca}^{2+}$-ATPase
\end{abstract}

\section{Cardiotonic agents in the treatment of congestive heart failure}

Congestive heart failure (CHF) is encountered during the final stages of various cardiovascular disorders, including cardiomyopathies, due to hypertensive, ischaemic or genetic origins or acute heart failure. In association with causal therapy to relieve the pathogenesis, attempts to ameliorate cardiac contractile dysfunction by means of cardiotonic agents, such as digitalis and catecholamines, had played a central role in the pharmacological therapy of CHF. Indeed, cardiotonic agents can effectively improve the quality of life (QOL) and exercise tolerance in most CHF patients. Since the early 1980s, extensive efforts have been made to develop novel cardiotonic agents to replace digitalis and catecholamines, because these agents have serious disadvantages such as arrhythmogenicity and unfavourable pharmacokinetic characteristics. During this time, it was believed that the improvement in QOL and exercise tolerance would lead to the subsequent amelioration of prognosis, including a

Correspondence: Professor $M$ Endoh, Department of Cardiovascular Pharmacology, Yamagata University School of Medicine, 2-2-2 lida-nishi, Yamagata, Japan.

E-mail: mendou@med.id.yamagata-u.ac.jp

Received 18 December 2006; accepted 22 December 2006; published online 26 February 2007 reduction in the morbidity and mortality of the patients. However, it has become evident that novel agents, such as amrinone and milrinone, exert unfavourable effects on the prognosis, even though these agents effectively improve the QOL of CHF patients. This realization has caused a large paradigm shift in pharmacotherapy from cardiotonic to cardioprotective therapy (Endoh, 2003).

\section{Mechanism of cardiotonic agents}

Clinically available cardiotonic agents, such as digitalis, catecholamines, amrinone and milrinone, act through an 'upstream' mechanism that is associated with an increase in the mobilization of intracellular $\mathrm{Ca}^{2+}$ in myocardial cells; thus, these agents are termed ' $\mathrm{Ca}^{2+}$ mobilizers', and they are associated with a risk of inducing $\mathrm{Ca}^{2+}$ overload resulting in cardiac arrhythmias, myocardial cell injury and, ultimately, cell death. In addition, a continuous increase in cardiac energy expenditure is supposed to lead to earlier exhaustion of myocardial cells. Thus, continuous administration of these agents, such as catecholamines and milrinone (a phosphodiesterase (PDE) III inhibitor), which elevate cellular cyclic AMP levels, may have energetic disadvantages due to 
$\mathrm{Ca}^{2+}$ overload and metabolic effects leading to apoptosis and arrhythmogenicity (Endoh et al., 1986).

Some agents that act directly on troponin $\mathrm{C}$ to increase its $\mathrm{Ca}^{2+}$ binding affinity ('central' mechanism) and/or on thin filament regulation of contractile proteins or directly on cross-bridge cycling ('downstream' mechanisms) are capable of exerting a positive inotropic effect (PIE) at an equivalent $\mathrm{Ca}^{2+}$ concentration; thus, these agents are termed ' $\mathrm{Ca}{ }^{2+}$ sensitizers' (Blinks and Endoh, 1986).

\section{$\mathrm{Ca}^{2+}$ sensitizers}

$\mathrm{Ca}^{2+}$ sensitizers are free from the risk of $\mathrm{Ca}^{2+}$ overload, and, in addition, they have energetic advantages in that they do not require an increase in activation energy such as that required for the increased $\mathrm{Ca}^{2+}$ transport induced by $\mathrm{Ca}^{2+}$ mobilizers in myocardial cells. Furthermore, $\mathrm{Ca}^{2+}$ sensitizers could improve haemodynamic parameters with a minimum increase in energy expenditure, even under pathological conditions, such as acidosis and stunned myocardium when $\mathrm{Ca}^{2+}$ mobilizers fail to increase myocardial contractility due to $\left[\mathrm{Ca}^{2+}\right]_{i}$-contractile protein uncoupling. $\mathrm{Ca}^{2+}$ sensitizers are further classified into three classes based on their mode of action: 'class I' agents act directly on troponin $\mathrm{C}$ to increase its $\mathrm{Ca}^{2+}$ binding affinity or stabilize the $\mathrm{Ca}^{2+}$-bound conformation of troponin C ('central' mechanism); 'class II' agents act on the thin filament complex, leading to increased $\mathrm{Ca}^{2+}$-binding affinity of troponin $\mathrm{C}$, or facilitate the thin filament regulation of the actin-myosin interaction ('downstream' mechanism); and 'class III' agents act directly on cross-bridge cycling, influencing a process beyond the regulatory site of $\mathrm{Ca}^{2+}$ and, therefore, could be termed 'cross-bridge enhancers' ('downstream' mechanism).

\section{Levosimendan}

Levosimendan was found in a screening for compounds that bind troponin $\mathrm{C}$ with high affinity. It is thought to bind to the $\mathrm{Ca}^{2+}$-saturated $\mathrm{N}$-terminal domain of troponin $\mathrm{C}$ and stabilize the troponin molecule with subsequent prolongation of its effect on contractile proteins (Endoh, 2003). In this issue of $\mathrm{Br} J$ Pharmacol, Louhelainen and co-workers examined the effectiveness of oral administration of levosimendan on the progression of CHF in hypertensive Dahl/ Rapp rats, focusing on the preventive effects of the compound on hypertension-induced cardiac remodelling and cardiomyocyte apoptosis (Louhelainen et al., 2007). Levosimendan exerted prominent beneficial effects in Dahl/ Rapp rats on a high-salt diet that developed severe hypertension, cardiac hypertrophy and moderate systolic dysfunction in the absence of treatment. Levosimendan (1) increased cardiac function; (2) improved survival; (3) ameliorated cardiac hypertrophy; (4) prevented cardiomyocyte apoptosis in a concentration-dependent manner; (5) normalized the salt-induced increase in the expression of atrial natriuretic peptide (ANP) and osteopontin mRNA; (6) lowered the plasma level of brain natriuretic peptide (BNP); (7) decreased the urinary excretion of noradrenaline; and (8) corrected the salt-induced decrease in the expression of SERCA2a and the SERCA2a/NCX ratio.

The important findings in this study are summarized as follows: long-term oral administration of levosimendan exerted beneficial effects on the chronic CHF caused by hypertensive cardiomyopathy in a Dahl/Rapp rat model, by improving haemodynamic function, suppressing remodelling and apoptosis, and normalizing genetic expression. These observations imply that the potential usage of oral levosimendan in the treatment of chronic CHF of certain aetiology should have favourable effects not only on QOL and exercise tolerance, but also on the long-term prognosis.

\section{Mechanisms of action of levosimendan}

The mechanisms of action of levosimendan that potentially contribute to the beneficial effects exerted in the heart failure model of Dahl/Rapp rats on a high-salt diet, appear to be rather complex, because levosimendan possesses actions other than increasing myofilament $\mathrm{Ca}^{2+}$ sensitivity. Based on previous studies, it is evident that levosimendan can act through at least three signalling pathways to elicit beneficial effects on the heart failure model: (1) it could act by increasing the $\mathrm{Ca}^{2+}$ sensitivity of contractile proteins by binding to troponin $\mathrm{C}$ (as a class $\mathrm{I} \mathrm{Ca}^{2+}$ sensitizer); (2) it could inhibit PDE III and (3) it might activate $\mathrm{K}_{\mathrm{ATP}}^{+}$channels in vascular smooth muscle cells and mitochondrial membranes. As a $\mathrm{Ca}^{2+}$ sensitizer, levosimendan has peculiar characteristics. Although the PIE of certain $\mathrm{Ca}^{2+}$ sensitizers, such as EMD 57033 and Org 30029, was not associated with an increase in $\mathrm{Ca}^{2+}$ transients (Endoh, 2002), detailed examination of the $\left[\mathrm{Ca}^{2+}\right]_{i}$-force relationship in the intact ventricular myocardium reveals that the PIE of levosimendan was associated with an increase in $\mathrm{Ca}^{2+}$ transients over a wide range of concentrations (Sato et al., 1998; Takahashi and Endoh, 2005). Furthermore, the PIE of levosimendan was abolished in the presence of the muscarinic receptor agonist carbachol, which has been shown to selectively inhibit a cyclic AMP-mediated pathway by inactivation of adenylyl cyclase via the inhibitory GTP-binding protein $\left(\mathrm{G}_{i}\right)$ and/or activation of phosphatases. Acidosis partially suppresses the PIE of levosimendan by decreasing the amplitude of $\mathrm{Ca}^{2+}$ transients, leaving its $\mathrm{Ca}^{2+}$ sensitizing action unaffected (Takahashi and Endoh, 2005). As levosimendan inhibits PDE III with high potency (Endoh, 2002), it is highly likely that the increase in $\mathrm{Ca}^{2+}$ transients can be ascribed to a moderate accumulation of cyclic AMP and that the PIE of levosimendan is elicited by a combination of a small increase in $\mathrm{Ca}^{2+}$ transients and its enhancement induced by troponin C-mediated $\mathrm{Ca}^{2+}$ sensitization (Endoh and Hori, 2006). However, whether or not the $\mathrm{Ca}^{2+}$ sensitizing effect of levosimendan is associated with an increase in $\mathrm{Ca}^{2+}$ transients in chronic CHF patients is still controversial. Thus, under pathophysiological conditions such as chronic $\mathrm{CHF}$, it is likely that the cyclic AMP-mediated pathway targeted by PDE III inhibition would be abolished and that the $\mathrm{Ca}^{2+}$ sensitizing action would become dominant compared with that in normal cardiac muscle (Hasenfuss et al., 1998). Could the anti-apoptotic effect and suppression of remodelling 
elicited by oral levosimendan in Dahl/Rapp rats be ascribed to the PIE of levosimendan? The answer is yes, but perhaps only partially. Suppression of the compensatory neurohumoral regulatory pathway, including sympathetic nerve activation, the renin-angiotensin-aldosterone system and cytokine production, by normalization of haemodynamic parameters mainly induced by the improvement in cardiac contractile dysfunction, may play a crucial role in the beneficial effects of levosimendan. In addition, levosimendan may have anti-apoptotic properties that are linked to activation of mitochondrial $\mathrm{K}_{\mathrm{ATP}}^{+}$channels (Kopustinskiene et al., 2001; Parissis et al., 2004). Furthermore, the contribution of peripheral vasodilatation to these effects, due to the opening of $\mathrm{K}^{+}$channels and the subsequent hyperpolarization of vascular smooth muscle cells, cannot be ignored as a factor that causes cardiac unloading. Whether the vasodilator effect of levosimendan is due to a direct effect of the compound binding to specific sites on $\mathrm{K}^{+}$channels, or partly mediated by the accumulation of cyclic AMP induced by inhibition of PDE III in vascular smooth muscle cells, remains unanswered in the current study.

\section{Future direction}

While the administration of cardiotonic agents for the treatment of heart failure is restricted to the aggravating phase of chronic CHF and cardiac pump insufficiency in acute $\mathrm{CHF}$, the beneficial effects of levosimendan on $\mathrm{CHF}$ due to hypertensive cardiomyopathy in Dahl/Rapp rats demonstrate the potential of oral levosimendan in the long-term treatment of chronic CHF. The beneficial effects of levosimendan might be elicited by the combination of a $\mathrm{Ca}^{2+}$ sensitizing action and additional actions that inhibit PDE III and activate $\mathrm{K}^{+}$channels. Large-scale clinical trials with this compound are ongoing; these are expected to unveil the clinical effectiveness of this compound in the treatment of chronic CHF patients. As chronic CHF in a clinical setting is much more complex than the CHF in the model used by Louhelainen et al. (2007), with inhomogenous aetiologies at different stages of severity, the final evaluation of the drug might be indecisive and equivocal (Endoh and Hori, 2006). Careful analysis of basic and clinical studies, taking into consideration any aspect that might influence the evaluation of levosimendan, will be required to determine the effectiveness of cardiotonic therapy, by means of $\mathrm{Ca}^{2+}$ sensitizers, in the treatment of chronic CHF.

\section{References}

Blinks JR, Endoh M (1986). Modification of myofibrillar responsiveness to $\mathrm{Ca}^{++}$as an inotropic mechanism. Circulation 73: III-85-III-98

Endoh M (2002). Mechanisms of action of novel cardiotonic agents. J Cardiovasc Pharmacol 40: 323-338.

Endoh M (2003). The therapeutic potential of novel cardiotonic agents. Expert Opin Investig Drugs 12: 735-750.

Endoh M, Hori M (2006). Acute heart failure: inotropic agents and their clinical uses. Expert Opin Pharmacother 7: 2179-2202.

Endoh M, Yanagisawa T, Taira N, Blinks JR (1986). Effects of new inotropic agents on cyclic nucleotide metabolism and calcium transients in canine ventricular muscle. Circulation 73: III-117-III-133.

Hasenfuss G, Pieske B, Castell M, Kretschmann B, Maier LS, Just H (1998). Influence of the novel inotropic agent levosimendan on isometric tension and calcium cycling in failing human myocardium. Circulation 98: 2141-2147.

Kopustinskiene DM, Pollesello P, Saris NE (2001). Levosimendan is a mitochondrial $\mathrm{K}_{\mathrm{ATP}}$ channel opener. Eur J Pharmacol 428: 311-314.

Louhelainen M, Vahtola E, Kaheinen P, Leskinen H, Merasto S, Kyto V et al. (2007). Effects of levosimendan on cardiac remodeling and cardiomyocyte apoptosis in hypertensive Dahl/Rapp rats. $\mathrm{Br} J$ Pharmacol 150: 851-861 (this issue).

Parissis JT, Adamopoulos S, Antoniades C, Kostakis G, Rigas A, Kyrzopoulos S et al. (2004). Effects of levosimendan on circulating pro-inflammatory cytokines and soluble apoptosis mediators in patients with decompensated advanced heart failure. Am J Cardiol 93: 1309-1312.

Sato S, Talukder MAH, Sugawara H, Sawada H, Endoh M (1998). Effects of levosimendan on myocardial contractility and $\mathrm{Ca}^{2+}$ transients in aequorin-loaded right-ventricular papillary muscles and indo-1-loaded single ventricular cardiomyocytes of the rabbit. J Mol Cell Cardiol 30: 1115-1128.

Takahashi R, Endoh M (2005). Dual regulation of myofilament $\mathrm{Ca}^{2+}$ sensitivity by levosimendan in normal and acidotic conditions in aequorin-loaded canine ventricular myocardium. Br J Pharmacol 145: 1143-1152. 